\title{
The Effect of Piezoelectric Fiber Rosette Configurations on Lamb Wave Direction Detection for Damage Localization
}

\author{
Shuai Jiang $\mathbb{D}^{1,2}$ Yiping Shen $\mathbb{D}^{1},{ }^{1}$ Songlai Wang $\mathbb{D},{ }^{1}$ Yanfeng Peng $\mathbb{D}^{1},{ }^{1}$ and Yi Liu $\mathbb{D}^{3}$ \\ ${ }^{1}$ Hunan Provincial Key Laboratory of Health Maintenance for Mechanical Equipment, Hunan University of Science and Technology, \\ Xiangtan 411201, China \\ ${ }^{2}$ Hunan Railway Professional Technology College, Zhuzhou 412000, China \\ ${ }^{3}$ Zhuzhou National Innovation Railway Technology Co., Ltd., Zhuzhou 412000, China
}

Correspondence should be addressed to Yiping Shen; yiping1011@163.com

Received 2 April 2021; Accepted 9 July 2021; Published 28 July 2021

Academic Editor: Yunze He

Copyright (c) 2021 Shuai Jiang et al. This is an open access article distributed under the Creative Commons Attribution License, which permits unrestricted use, distribution, and reproduction in any medium, provided the original work is properly cited.

\begin{abstract}
Piezoelectric fiber rosettes respond to the directivity characteristics of Lamb waves, and therefore, are useful in detecting the Lamb wave propagation direction. Considering material damage as a secondary wave source, two piezoelectric fiber rosettes are arranged to measure the scattered wave propagation directions for damage localization. The influences of various rosette configurations, i.e., $45^{\circ}$-rectangular, $135^{\circ}$-rectangular, $60^{\circ}$-delta, and $120^{\circ}$-delta, on the estimation accuracy of the propagation direction are investigated in this paper. The response of the piezoelectric fiber to the $A_{0}$ mode Lamb wave under narrowband tone-burst excitation is theoretically derived. Experimental tests and piezoelectric coupling simulations are performed to obtain the Lamb wave signal of each fiber. The matching pursuit (MP) algorithm is applied to extract the weak damage-related wave packet by using Hann-windowed narrowband excitation as an atom. The Lamb wave propagation directions are estimated based on the error function. The accuracies of the directions with 4 types of rosette configurations are compared, and their error sources are discussed. The results show that the accuracy of the $135^{\circ}$-rectangular configuration is relatively satisfactory, and the errors depend on the size and location of each fiber in the rosette. The proposed damage localization method is validated by experimental tests. The predicted locations are close to the actual damage location. The research results are significant for piezoelectric fiber rosette design and optimization and damage location without wave speed or time-of-flight information in complex or irregular structures.
\end{abstract}

\section{Introduction}

Lamb-wave-based damage detection in plate-like structures draws increasing attention as Lamb waves can travel a long distance even in materials with low attenuation and are highly susceptible to small damage along a propagation path $[1,2]$. For isotropic plates, damage can be located after detecting the scattered Lamb wave signal by at least three sensors and applying conventional time-of-flight triangulation. However, the approach requires a priori knowledge of the Lamb wave velocity in a plate to translate arrival time measurements into damage locations. This requirement is a fundamental limitation for complex or irregularly shaped structures.
An alternative damage localization technique is to apply a rosette-like directional sensor to predict the wave direction [3-6]. These directional sensors are similar with wellknown electrical resistance strain gage rosette constructed of three gage grids in a certain configuration, which are generally used to resolve the principal strain directions. Thus, the wave propagation direction can be evaluated from the principal strain direction when this direction coincides with the principal strain direction for isotropic plates. Consequently, damage can be located from the point of the intersection of two wave propagation directions obtained from two directional sensors. Two directional sensor types have been proposed: the first sensor is based on fiber optics [7], and the second sensor is based on different piezoelectric elements, 
e.g., macrofiber composites (MFCs) $[8,9]$, metal-core piezoelectric fibers [10], rectangular piezoelectric sheets [11], and round piezoelectric fibers [12]. The directivities of the piezoelectric element responses to Lamb waves have been well explored, which enables the use of rosettes for wave direction evaluation.

Kundu et al. [4] proposed a technique that performs acoustic source localization by acquiring and analyzing the signal data at several sensors in L-shaped clusters. The wave propagation direction (of the group velocity) is determined by the time difference of the arrival of waves at each sensor of a cluster. Their method was subsequently extended by Yin et al. [13, 14], and different Z-shaped clusters were introduced to decrease the number of required sensors. This method can works for an anisotropic plate despite the wave direction does not coincide with the principal strain direction [15]. However, it is difficult to extract the small differences in the time of arrival (TOA) from the weak noise signal of each sensor.

Three-element rectangular or delta rosettes are preferable in application where the principal strains are unknown $[16,17]$. Rosette configurations allow us to determine the principal strain direction of the Lamb wave. The $120^{\circ}$ and $60^{\circ}$ delta configurations are applied in Refs. [7-10] to locate acoustic sources and $45^{\circ}$ rectangular rosette in Refs. [11, 12]. The high directivity of those piezoelectric elements to sensing Lamb waves contributes to the principal strain direction evaluation. The delta configuration has the advantage that it enables a somewhat simpler estimation when the directivity of the rosette sensor is approximated by a cosine-squared function, as in Refs. [7, 10]. However, the presumed directivity function is not adapted to highfrequency excitation conditions. In conclusion, the rosette configuration is an important parameter for the accuracy of Lamb wave direction estimation but is still not sufficiently characterized.

The primary focus of this paper is the piezoelectric fiber rosette configurations; these rosettes are used to determine the damage location based on the measured scattered wave propagation directions. Four types of well-established rectangular and delta rosette configurations of conventional electrical strain gauges are discussed, i.e., the $45^{\circ}$ and $135^{\circ}$ rectangular configurations and the $60^{\circ}$ and the $120^{\circ}$ delta configurations. Considering the damping effect, the directivity response of the piezoelectric fiber to $A_{0}$ mode Lamb wave is theoretically derived, which allows the Lamb wave propagation direction to be evaluated. As the damage is small and can be treated as a secondary wave source, the damage location is determined by the intersection of the scattered wave propagation directions with two rosettes. Coupled finite element analysis and experimental tests are performed to demonstrate the accuracies of Lamb wave propagation direction estimations with various rosette configurations. The matching pursuit (MP) algorithm is applied to extract the incident and the scattered wave signal from the measured noisy Lamb wave signals by using Hann-windowed narrowband excitation as a so-called atom. Error analyses for Lamb wave direction estimations are discussed in terms of various rosette configurations.
The performance of the rosettes for damage localization is validated through artificial damage manufactured on the specimen.

\section{Damage Localization Method with Directional Piezoelectric Fiber Rosettes}

Assume that piezoelectric fiber is well bonded to the top surface of a plate with thickness of $2 h$, as shown in Figure 1 . The angle between the wave propagation direction $x^{\prime}$ and the lengthwise direction of piezoelectric fiber is defined as $\theta$. The response voltage amplitude depends on the angle $\theta$ between the wave propagation direction $x^{\prime}$ and the lengthwise direction of piezoelectric fiber. The directional response of piezoelectric fiber under narrowband tone-burst excitation has been theoretically deduced in our previous work [12]. To investigate the effect of piezoelectric fiber rosette configurations on Lamb wave direction detection, the lengths and the actual positions of piezoelectric fibers are considered. Therefore, the wave attenuation factors, including geometry spreading and material damping, are considered in this paper. The in-plate displacement of the flexural Lamb wave can be written as [8]

$$
\begin{aligned}
u_{x^{\prime} \mid x^{\prime}=L}= & B k \sqrt{\frac{r_{a}}{L}}\left(\frac{\sinh a z}{\cosh a h}-\frac{2 a b}{k^{2}+b^{2}} \cdot \frac{\sinh b z}{\cosh b h}\right) \\
& \times e^{-k_{d}\left(L-r_{a}\right)+i(k L-\omega t-\pi / 2)},
\end{aligned}
$$

where $L$ is the distance between the Lamb wave source point and the center of the piezoelectric fiber, $B$ is an arbitrary constant, $r_{a}$ is the radius of the actuator, $k_{d}$ is the attenuation factor in the material, $h$ is half of the plate thickness, and the parameters $a$ and $b$ are defined as

$$
a=\sqrt{k^{2}-\frac{\omega^{2}}{c_{L}^{2}}}, b=\sqrt{k^{2}-\frac{\omega^{2}}{c_{T}^{2}}}, k=\frac{2 \pi}{\lambda},
$$

where $k, \lambda$, and $\omega$ are the wavenumber, wavelength, and circular central frequency, respectively, and $c_{L}$ and $c_{T}$ are the longitudinal velocity and transversal velocity, respectively, in the plate.

Similarly, the piezoelectric fiber's response to the flexural Lamb wave is expressed as [12]

$$
\bar{V}=\frac{E d_{33} \lambda}{\pi e_{33}} \sqrt{\frac{r_{a}}{L}} e^{-k_{d} L} S(\theta) \times \bar{\varepsilon}_{x^{\prime} x^{\prime}},
$$

where $\bar{\varepsilon}_{x^{\prime} x^{\prime}}$ is the amplitude of the in-plane strain under excitation and $S(\theta)$ is the sensitivity factor

$$
S(\theta)=\cos \theta \sin \left(\frac{\pi l \cos \theta}{\lambda}\right) .
$$

Hann-windowed narrowband excitation signals are typically employed in applications to excite a Lamb wave, which is expressed as 


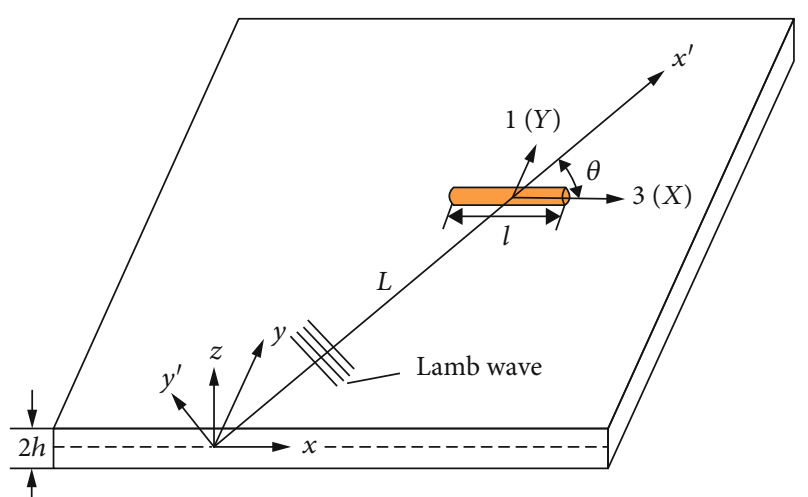

FIGURE 1: Directional response of a piezoelectric fiber to Lamb wave.

$A(t)=A_{a}\left[H(t)-H\left(t-\frac{2 \pi n}{\omega_{c}}\right)\right] \times \sin \left(\omega_{c} t\right)\left(1-\cos \left(\frac{\omega_{c} t}{n}\right)\right)$,

where $A_{a}$ is the excitation amplitude, $n$ is the cycle number of the tune-burst excitation signal, and $\omega_{c}$ is the central circular frequency.

The response to the narrowband excitation can be expressed as [12]

$$
\begin{aligned}
U(t) & =\int_{\omega_{c}-\Delta \omega / 2}^{\omega_{c}+\Delta \omega / 2} A(\omega) \bar{V}(\omega) e^{-i \omega t} d \omega \\
& =\int_{\omega_{c}-\Delta \omega / 2}^{\omega_{c}+\Delta \omega / 2} \frac{E d_{33} \lambda}{\pi e_{33}} \sqrt{\frac{r_{p}}{L}} e^{-k_{d} L} S(\theta) \bar{\varepsilon}_{x^{\prime} x^{\prime}} A(\omega) e^{-i \omega t} d \omega,
\end{aligned}
$$

where $\Delta \omega=4 \omega_{c} / n$ denotes the frequency bandwidth.

From Equation (6), the time-domain response to the narrowband excitation depends on the angle $\theta$ and the distance $L$. Considering Equation (6), the response voltage is just equal to the excitation with a time shift, a phase variation, and additional amplitude attenuation. The response voltages can be represented by their Hilbert envelope for disregarding the effect of the phase change with in the wave packet [18], and the scattered wave peak of the energy envelopes is introduced to quantify the response amplitude, which is expressed as

$$
\tilde{U}=|U(t)+i H[U(t)]|_{\text {peak }}
$$

where $H[U(t)]$ denotes the Hilbert transform (HT) of the wave signal.

Let three piezoelectric fibers $A, B$, and $C$ are arranged in an arbitrary rosette configuration, as shown in Figure 2(a), and $\alpha_{i}(i=A, B, C)$ denote the angle between the $i^{\text {th }}$ piezoelectric fiber and the referenced piezoelectric fiber $A$. It is assumed that $\alpha_{A}$ is 0 . According to Equation (7), the voltage response of the three piezoelectric fibers can be expressed as

$$
\tilde{U}_{i}=\tilde{U}_{\max } \cos \left(\theta-\alpha_{i}\right) \sin \left(\frac{\pi l \cos \left(\theta-\alpha_{i}\right)}{\lambda}\right) \quad i=A, B, C,
$$

where $\tilde{U}_{i \max }$ is the maximum voltage response of the piezoelectric fiber, which is parallel to the Lamb wave propagation direction.

In this paper, the actual sum is still applied to normalize the response. The normalized amplitudes of piezoelectric fibers can be expressed as

$$
T_{i}=\frac{3 \tilde{U}_{i}}{\sum_{i=1}^{3} \tilde{U}_{i}}=\frac{3 \cos \left(\theta+\alpha_{i}\right) \sin \left(\pi l \cos \left(\theta+\alpha_{i}\right) / \lambda\right)}{\sum_{i=1}^{3} \cos \left(\theta+\alpha_{i}\right) \sin \left(\pi l \cos \left(\theta+\alpha_{i}\right) / \lambda\right)} .
$$

The angle $\theta$ can be evaluated by the error between the experimental normalized voltage amplitude and the theoretical normalized voltage amplitude using the numerical computation method. The error is defined as [12]

$$
e(\widehat{\theta})=\sqrt{\frac{1}{3} \sum_{i=1}^{3}\left(T_{i}-T_{i}(\theta \wedge)\right)^{2}}
$$

where $\widehat{\theta}$ is the estimation of the Lamb wave propagation direction for theoretical calculation.

According to Equation (10), the error value $e(\widehat{\theta})$ will be 0 when $\widehat{\theta}=\theta$. In practical application, $e(\widehat{\theta})$ is impossible to be 0 because of the unavoidable measurement error. Therefore, the estimation of $\theta$ is assumed to be $\widehat{\theta}$ when $e(\widehat{\theta})$ trends to the minimum. The detailed discussion is presented in our previous work [12]. Considering the damage as a secondary wave source, the damage location can be evaluated by the intersection of the scattered wave propagation directions by two rosettes, as shown in Figure 2(b). The damage location $(x, y)$ can be determined by the scattered wave propagation directions $\theta_{1}$ and $\theta_{2}$ according to

$$
\left\{\begin{array}{l}
x=\frac{y_{2}-y_{1}+x_{1} \tan \theta_{1}-x_{2} \tan \theta_{2}}{\tan \theta_{1}-\tan \theta_{2}} \\
y=\left(x-x_{1}\right) \tan \theta_{1}+y_{1}
\end{array}\right.
$$

where $\left(x_{1}, y_{1}\right)$ and $\left(x_{2}, y_{2}\right)$ are the coordinates of the layout origin of the corresponding rosette.

Considering the dimension and the configuration of the piezoelectric rosette, the actual direction angle is different from the theoretical angle of the rosette. In theory, the voltage responses of the piezoelectric fibers $B$ and $C$ are referenced to the piezoelectric fiber $A$ as the base, and the distance error $\Delta L_{i}$ and the angle error $\Delta \theta_{i}$ are included in Equation (8). Figure 3 shows the distance error $\Delta L_{B}$ and the angle error $\Delta \theta_{B}$ of the piezoelectric fiber $B$. Therefore, Equation (8) is rewritten as

$$
\tilde{U}_{i}=\tilde{U}_{i \max } \cos \left(\theta-\alpha_{i}+\Delta \theta_{i}\right) \sin \left(\frac{\pi l \cos \left(\theta-\alpha_{i}+\Delta \theta_{i}\right)}{\lambda}\right),
$$

where $U_{i \max }$ is the maximum voltage response of the piezoelectric fiber which is located in parallel with the Lamb wave propagation direction at the distance $L+\Delta L_{i}$, while $U_{i \max }$ 


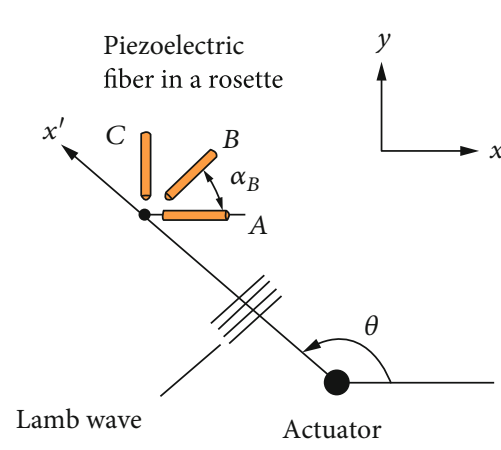

(a)

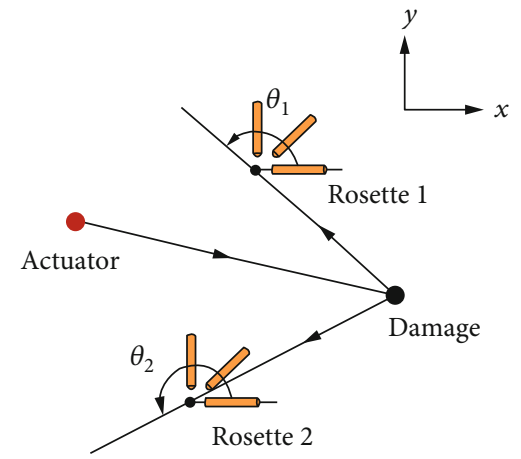

(b)

FIGURE 2: Damage location with the estimated scattered wave directions. (a) The Lamb wave propagation direction. (b) Damage location with two rosettes.
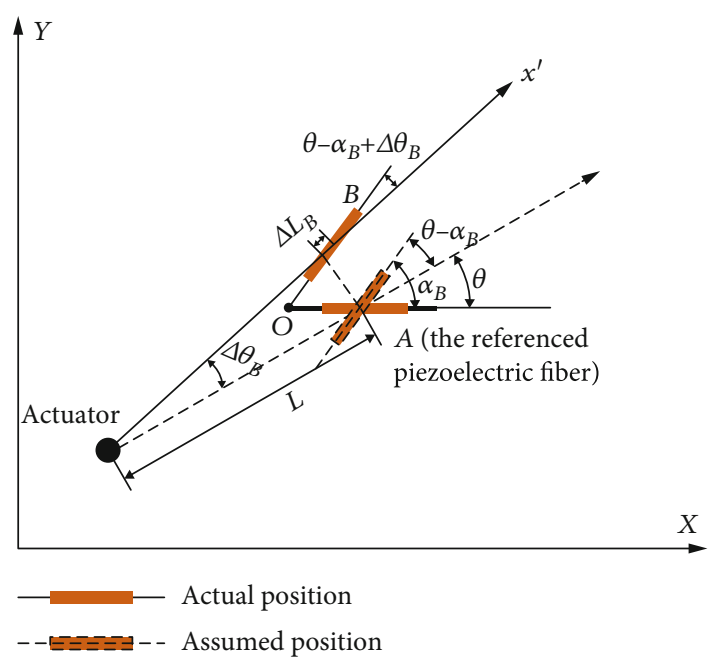

Figure 3: The error of the direction estimation with the rosette.

used in Equation (8) is the response of the piezoelectric fiber located at the distance $L$.

Similar to conventional electrical strain gauges, piezoelectric fiber rosettes can be arranged with different configurations. Four types of rosette configurations are discussed in this paper, as shown in Figure 4. Three piezoelectric fibers are numbered in the counterclockwise direction. $O$ is the layout origin for rosette sensor placement. Figure 4(a) shows the $45^{\circ}$ rectangular configuration denoted by $\mathrm{RC} 1$ in this paper, and the configuration angles of the three piezoelectric fibers are $0^{\circ}, 45^{\circ}$, and $90^{\circ}$, respectively. Figure 4 (b) shows the $135^{\circ}$ rectangular configuration denoted $\mathrm{RC} 2$, and the configuration angles of the three piezoelectric fibers are $0^{\circ}, 135^{\circ}$, and $90^{\circ}$, respectively. Figure 4 (c) shows the $60^{\circ}$ delta configuration denoted by $\mathrm{DC} 1$, and the configuration angles of the three piezoelectric fibers are $0^{\circ}, 120^{\circ}$, and $240^{\circ}$, respectively. Figure $4(\mathrm{~d})$ shows the $120^{\circ}$ delta configuration denoted by DC2, and the configuration angles are the same with DC1. In this paper, the piezoelectric fiber with a length of $10 \mathrm{~mm}$ is cut from a round piezoelectric fiber with a length of $150 \mathrm{~mm}$ and a diameter of $0.8 \mathrm{~mm}$, produced by Smart Material Corp. The piezoelectric material is PZT SP505 (Navy type II). The electrodes at two ends of a piezoelectric fiber are covered with silver paint. The signal wires are wired to the electrodes of the piezoelectric fibers and connected to the signal collector.

\section{Experiment Test and Simulation Analysis}

The accuracies of the Lamb wave direction estimations using the four different rosette configurations are compared by experimental tests and finite element simulations with coupled-field elements. The damage localization method is validated by experimental tests with artificial damage.

3.1. Experimental Test Setup. Rectangular aluminum plate specimens are employed with dimensions of $1 \mathrm{~m} \times 1 \mathrm{~m}$ and a thickness of $1 \mathrm{~mm}$ for both simulation analysis and experimental testing. The density is $2730 \mathrm{~kg} / \mathrm{m}^{3}$, the elastic modulus is $68.9 \mathrm{GPa}$, and Poisson's ratio is 0.33 . A piezoelectric wafer is applied to excite the Lamb wave in the plate. This wafer is made from PZT8 material with a radius of $10 \mathrm{~mm}$ and a thickness of $0.8 \mathrm{~mm}$. The piezoelectric wafer is manufactured by Smart Material Corp., USA. A 5-cycle narrowband toneburst signal modulated by the Hamming window is employed to excite a Lamb wave in the plate. An EPA-10 power amplifier, which is produced by Piezo System Inc., USA, is applied to amplify the excitation narrowband signals. An $80 \mathrm{~V}$ peak-to-peak amplification excitation is applied to the actuator. An NI PXle-6361 platform is applied to collect the response output voltage of the piezoelectric sensors.

The piezoelectric fiber rosettes are directly connected to the platform to acquire the electric signals generated through the piezoelectric coupling between the strain field and the electric field. Four piezoelectric fiber rosette configurations are applied to estimate the incident Lamb wave propagation direction, as shown in Figure 5(a). The actuator is placed at an angle of $32^{\circ}$ with four rosettes, and the distance between the actuator and the sensors is $300 \mathrm{~mm}$. For the damage location experimental test, a hole with a diameter of $20 \mathrm{~mm}$ is manufactured in the aluminum plate. The arrangement of the actuator, rosettes, and damage is shown in Figure 4(b). The central frequencies of the excitation waves are $20 \mathrm{kHz}$, $40 \mathrm{kHz}$, and $60 \mathrm{kHz}$. The sampling frequency is $2 \mathrm{MHz}$. The measured signals of the piezoelectric sensors are averaged 


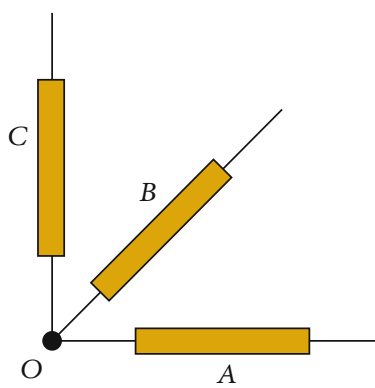

(a)

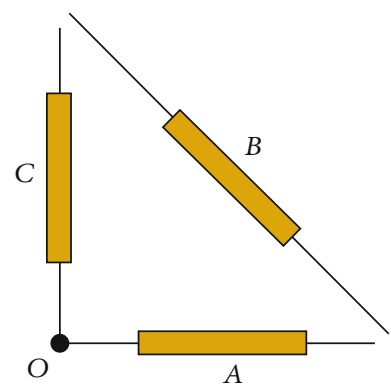

(b)

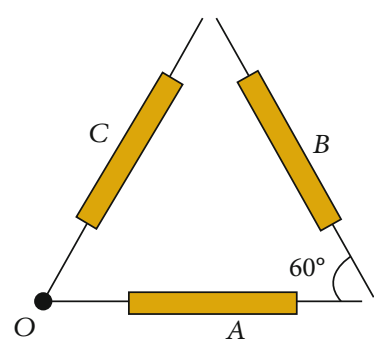

(c)

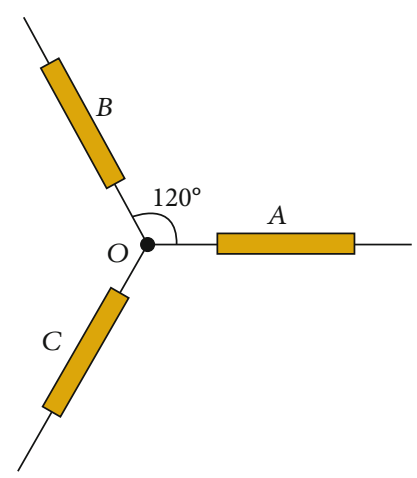

(d)

FIGURE 4: Four types of piezoelectric fiber rosette configuration: (a) rectangular (RC1); (b) rectangular (RC2); (c) delta (DC1); and (d) delta (DC2).

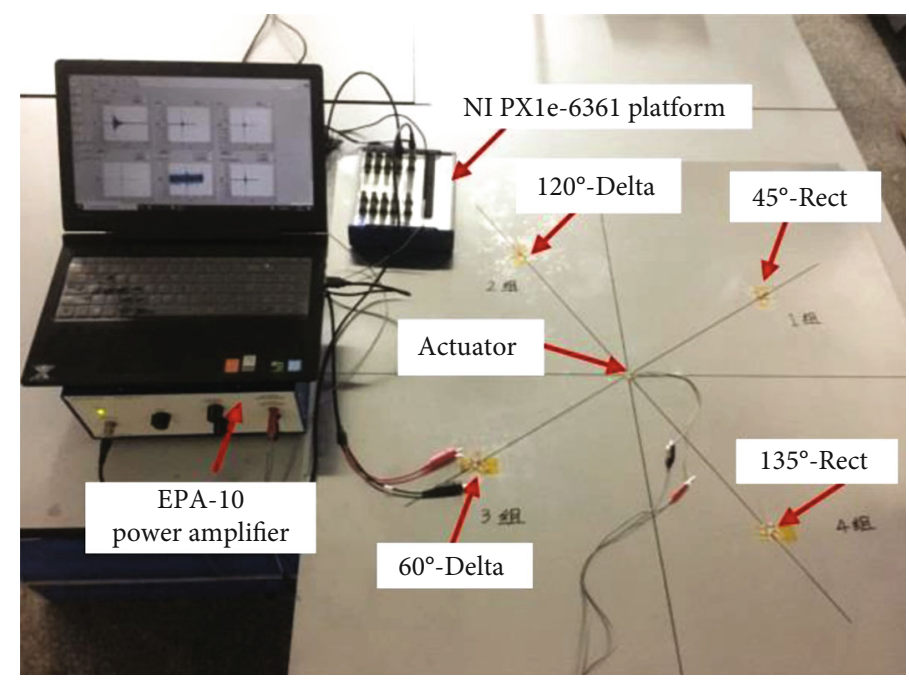

(a)

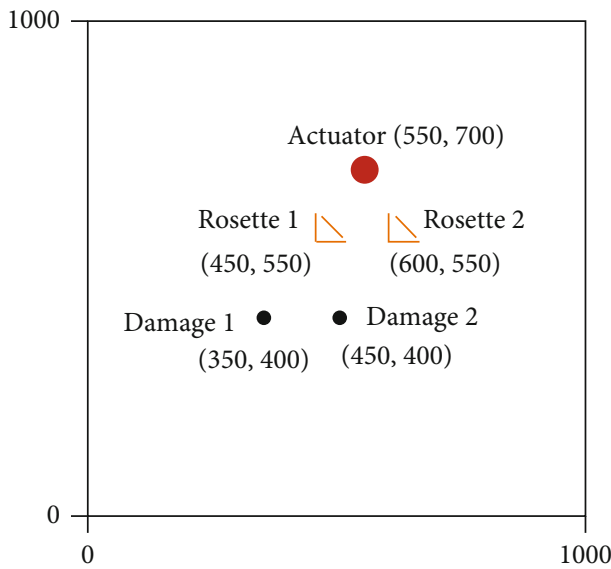

(b)

Figure 5: Experimental test setup. (a) Four rosette configurations. (b) Damage location.

100 times. An NI LabVIEW program was written to generate excitation waves and acquire the sensor data. A MATLAB program was written for Lamb wave signal analysis, Lamb wave propagation direction estimation, and damage location computation.

3.2. Coupled Finite Element Analysis. Mechanical-electrical coupled finite element analysis, which takes into account both the piezoelectric wafer actuator and the piezoelectric fibers, is performed. The corresponding material physical parameters are listed in Table 1.

The commercial finite element software ANSYS is employed for this analysis. SOLID5 coupled-field elements are applied to simulate the piezoelectric effects of the piezoelectric wafer and piezoelectric fibers. SHELL181 elements are employed to model the aluminum plate specimen. The mesh size of the finite element model is $1 \mathrm{~mm}$ which is smaller than one-twentieth of the Lamb wavelength at $60 \mathrm{kHz}$ to ensure the accuracy of the analysis results. The time step is set to $0.5 \mu \mathrm{s}$. Both the mesh size and the time step set satisfy the criteria of transient dynamic analysis [19]. A total of 1,033,000 elements are employed. The voltage DOFs of the nodes located on two surfaces of the piezoelectric wafer and two ends of each piezoelectric fiber are coupled to only one master node to simulate their electrodes, as shown in Figure 6.

The excitation voltage of the narrowband tone-burst signal is applied to the upper electrode of the piezoelectric wafer. The output voltages of the three piezoelectric fibers in the rosettes are analyzed to calculate the Lamb wave propagation direction. To verify the effects of the rosette configurations on the accuracies of the direction estimation, the first arrival flexural Lamb waves are employed to extract the response amplitudes of each piezoelectric fiber. The resultant voltage outputs of the piezoelectric fiber $C$ in RC2 at a central frequency of $40 \mathrm{kHz}$ are plotted in Figure 7, which is located in Rosette 1 to measure the scattered wave from damage 1 . The first arrival wave of the simulation resultant wave shows agreement with the experimental measured signal, and an amplitude difference is caused by the measurement noise. Note that the obscurity of the scattered waves is attributed to their weakness. Considering the overlapped wave packets 
TABLe 1: Material physical parameters of the piezoelectric wafer and piezoelectric fibers.

\begin{tabular}{lcc}
\hline Parameter & Piezoelectric wafer & Piezoelectric fibers \\
\hline Density $\left(\mathrm{kg} / \mathrm{m}^{3}\right)$ & 7600 & 7850 \\
Relative dielectric constant & 900 & 1850 \\
Piezoelectric constant $d_{33}\left(\times 10^{-12} \mathrm{C} / \mathrm{N}\right)$ & 225 & 440 \\
Piezoelectric constant $d_{31}\left(\times 10^{-12} \mathrm{C} / \mathrm{N}\right)$ & 97 & 185 \\
Elastic compliance constant $s_{11}^{E}\left(\times 10^{-12} \mathrm{~m} / \mathrm{N}\right)$ & 11.20 & 18.50 \\
Elastic compliance constant $s_{33}^{E}\left(\times 10^{-12} \mathrm{C} / \mathrm{N}\right)$ & 13.36 & 20.70 \\
Electromechanical coupling factors $k_{p}$ & 0.6 & 0.62 \\
Electromechanical coupling factors $k_{33}$ & 0.7 & 0.72
\end{tabular}

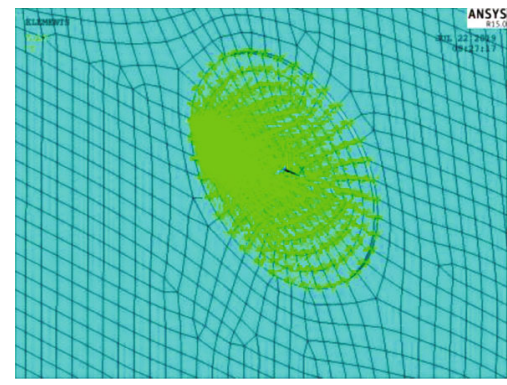

(a)

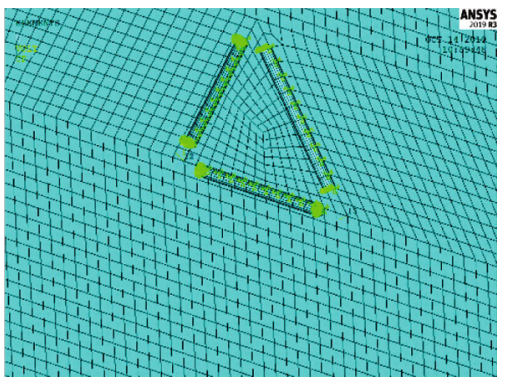

(b)

FIGURE 6: Coupled analysis model. (a) Local mesh of piezoelectric wafer. (b) Local mesh of RD1.

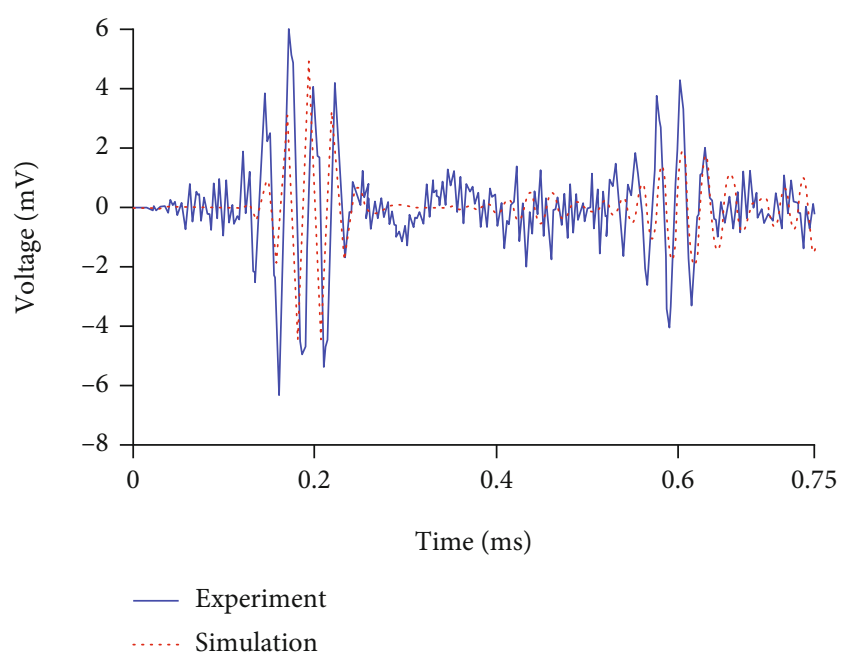

Figure 7: Resultant voltage outputs of the piezoelectric fiber $C$ in $\mathrm{RC} 2$ at $40 \mathrm{kHz}$.

and the measurement noise, extraction of the damage-related wave packets from the measured signal is difficult. Therefore, considering a suitable signal decomposition method is essential to effectively separate the individual wave packets and obtain the exact required wave for further scattered wave direction calculation.

3.3. Signal Decomposition Based on the MP Algorithm. More advanced signal processing techniques are required to accurately separate the weak scattered wave packet from the noisy overlapped signal in an application. Sparse reconstruction has attracted a substantial amount of attention in ultrasonic guided wave-based damage detection $[18,20]$. The MP algorithm introduced by Mallat and Zhifeng [21] is one of the most extensively applied algorithms for sparse signal representation. MP is an iterative greedy algorithm that computes an accurate solution for a signal in terms of the linear combinations of predefined atoms that construct an overcomplete dictionary. The algorithm of MP is described as follows.

Step 1. Construct a dictionary $\mathbf{D}$

$$
\mathbf{D}=\left\{g_{\gamma_{1}}, g_{\gamma_{2}}, \cdots, g_{\gamma_{j}}, \cdots g_{\gamma_{J}}\right\}
$$

where $\gamma_{j}$ is the $j^{\text {th }}$ parameter set of possible parameter combinations and $g_{\gamma_{j}}$ is the atom determined by the $j^{\text {th }}$ parameter set.

Step 2. Initialize the iteration number $\kappa=1$ and set the measured signal $f$ to the residual $R^{\kappa}$.

Step 3. Search for the dictionary atom $g_{\gamma_{\kappa}}$ that best resembles the measured signal $f$, which is achieved by solving the optimization problem

$$
g_{\gamma_{\kappa}}=\arg \max _{g_{\gamma_{j}} \in \mathbf{D}}\left|\left\langle R^{\kappa}, g_{\gamma}\right\rangle\right| \text {. }
$$

Step 4. Calculate the amplitude of the chosen atom to the measured signal 


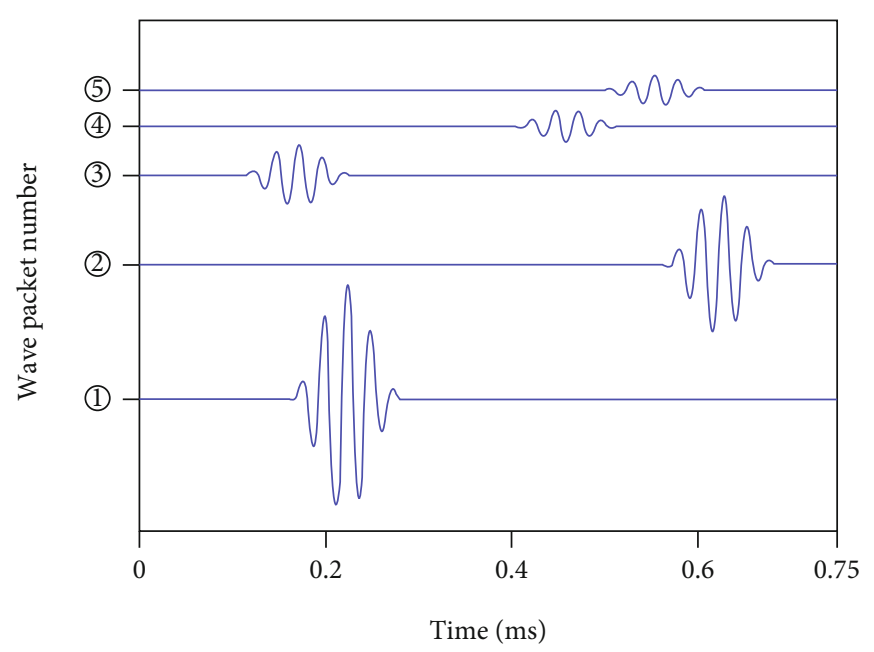

(a)

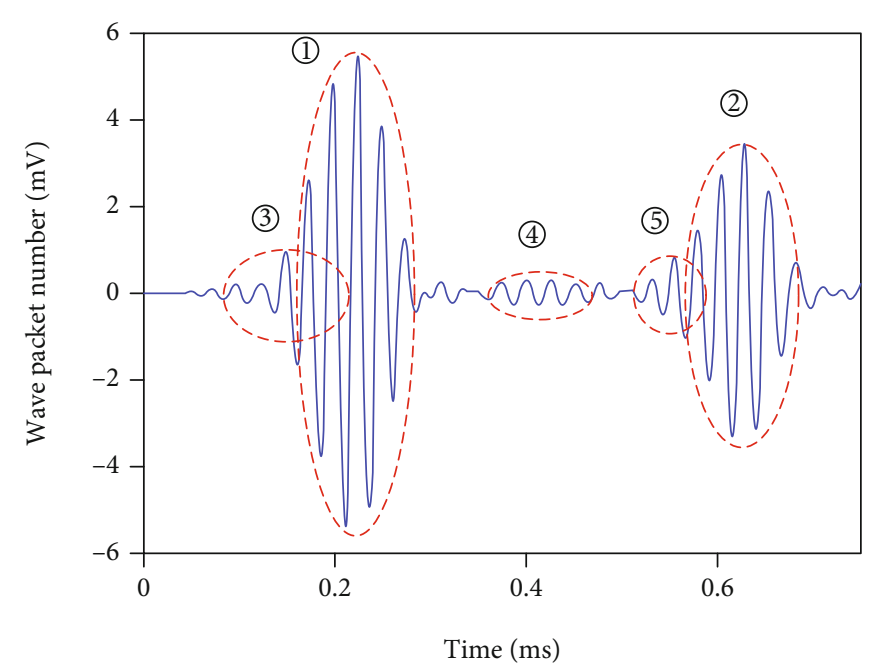

(b)

FIGURE 8: Wave packets decomposed after five iterations using the MP algorithm. (a) MP decomposition results and (b) reconstructed signal.

$$
c_{\kappa}=\left\langle R^{\kappa}, \tilde{g}_{\gamma_{\kappa}}\right\rangle
$$

where $\tilde{g}_{\gamma_{\kappa}}=g_{\gamma_{\kappa}} /\left\langle g_{\gamma_{\kappa}}, g_{\gamma_{\kappa}}\right\rangle$.

Step 5. Calculate a new residue by subtracting the chosen atom and adjusting the amplitude

$$
R^{\kappa+1}=R^{\kappa}-c_{\kappa} g_{\gamma_{\kappa}}
$$

Step 6. Perform the next iteration with the residual signal $R^{\kappa+1}$, i.e., return to Step 3, until the energy of the residual signal becomes sufficiently small. After $K$ iterations, MP decomposes the signal into

$$
f_{\text {recon }}=\sum_{i=1}^{K} c_{\kappa} g_{\gamma_{k}}+R^{K+1}
$$

To quickly and effectively decompose the measured signal, many possible atom functions are employed to construct an overcomplete dictionary, such as the Gabor atom [22], Chirplet atom [23], and an atom based on Hann-windowed narrowband excitation $[18,24,25]$. The atom dictionary should take into account the actual problem. Equations (1) and (6) indicate that the measured signal of each piezoelectric fiber is equal to the excitation with a time shift, a phase variation, and additional amplitude attenuation. Therefore, the atom in the overcomplete waveform dictionary is defined as [18]

$$
\begin{aligned}
g_{\gamma}(t)= & {\left[H(t-\tau)-H\left(t-\tau-\frac{2 \pi n}{\omega_{c}}\right)\right] \times \sin \left(\omega_{c}(t-\tau)+\varphi\right) } \\
& \cdot\left(1-\cos \left(\frac{\omega_{c}(t-\tau)}{n}\right)\right),
\end{aligned}
$$

where $\tau$ is the time delay and $\varphi$ is the shifted phase.
The finite set of parameters $\tau, \phi$, and $\omega_{c}$ for the dictionary should be discretized uniformly for the measured Lamb wave signal [26]. $\tau$ is discretized as $n T_{s}$, where $n$ and $T_{s}$ denote the sample length and the sampling rate, respectively. $\omega_{c}$ is the excitation central frequency. Many optimization algorithms can be applied to determine the parameter set, such as the genetic algorithm (GA) [27] and the artificial bee colony algorithm [28]. The GA is employed in this paper to obtain the global optimal solution in a continuous parameter space.

The damage-scattered Lamb wave signal with an excitation frequency $40 \mathrm{kHz}$, as shown in Figure 8, is decomposed after five iterations by applying the proposed MP algorithm, which is based on GA optimization. Figure 8(a) represents the individual wave packet after decomposing the measured signal, and Figure $8(\mathrm{~b})$ is the reconstructed signal. Wave packets (1) and (3) are the first-arrival A0 and S0 waves, respectively, (4) is the damage-scattered wave, and (2) and (5) are bounced back from the edge. The dictionary is based on Hann-windowed narrowband excitation as an atom, which can match the individual wave packet and the weak scattered wave packet. The MP method has the advantage of excellent noise robustness.

\section{Results and Discussion}

4.1. Lamb Wave Direction Estimation Results and Error Analysis. Lamb wave direction estimation results of the four types of rosette configurations are listed in Table 2. Comparing the results of four rosette configurations from simulation and experiment signals, ANSYS results indicate that the estimation errors increased as the excitation frequency increased; the rosette in RC2 shows the best estimation accuracy, which is less than 4\%; DC2 shows the largest error of 15\%; and RC1 and DC1 have an equivalent accuracy of less than $8 \%$. Experimental test results show larger errors than the ANSYS results. RC2 and DC1 have equivalent accuracies, which are less than $8 \%$; RC1 presents a larger error than $10 \%$, and DC2 shows the largest error of $30 \%$. The differences 
TABLE 2: Lamb wave direction estimation of the four types of rosette configurations.

\begin{tabular}{|c|c|c|c|c|c|c|c|c|c|}
\hline \multirow{2}{*}{ Frequency $(\mathrm{kHz})$} & \multirow{2}{*}{ Results } & \multicolumn{2}{|c|}{$\mathrm{RC} 1$} & \multicolumn{2}{|c|}{$\mathrm{RC} 2$} & \multicolumn{2}{|c|}{$\mathrm{DC} 1$} & \multicolumn{2}{|c|}{ DC2 } \\
\hline & & $1^{\circ}$ & $/ \% \mathrm{E}$ & $1^{\circ}$ & $1 \% \mathrm{E}$ & $1^{\circ}$ & $1 \% \mathrm{E}$ & $1^{\circ}$ & $1 \% \mathrm{E}$ \\
\hline 20 & ANSYS & 32.3 & 0.8 & 32.0 & -0.2 & 30.2 & -5.6 & 33.3 & 3.9 \\
\hline 40 & ANSYS & 33.3 & 3.9 & 33.1 & 3.3 & 32.6 & 1.8 & 36.4 & 13.8 \\
\hline 60 & ANSYS & 30.3 & -5.5 & 32.3 & 0.8 & 34.8 & 7.8 & 36.8 & 15.0 \\
\hline 20 & Test & 37.8 & 18.1 & 32.4 & 1.3 & 33.7 & 5.2 & 42.4 & 32.5 \\
\hline 40 & Test & 36.2 & 13.1 & 31.4 & -1.9 & 33.1 & 3.3 & 42.9 & 33.9 \\
\hline 60 & Test & 34.0 & 6.3 & 30.0 & -6.3 & 34.6 & 8.1 & 41.9 & 30.8 \\
\hline
\end{tabular}

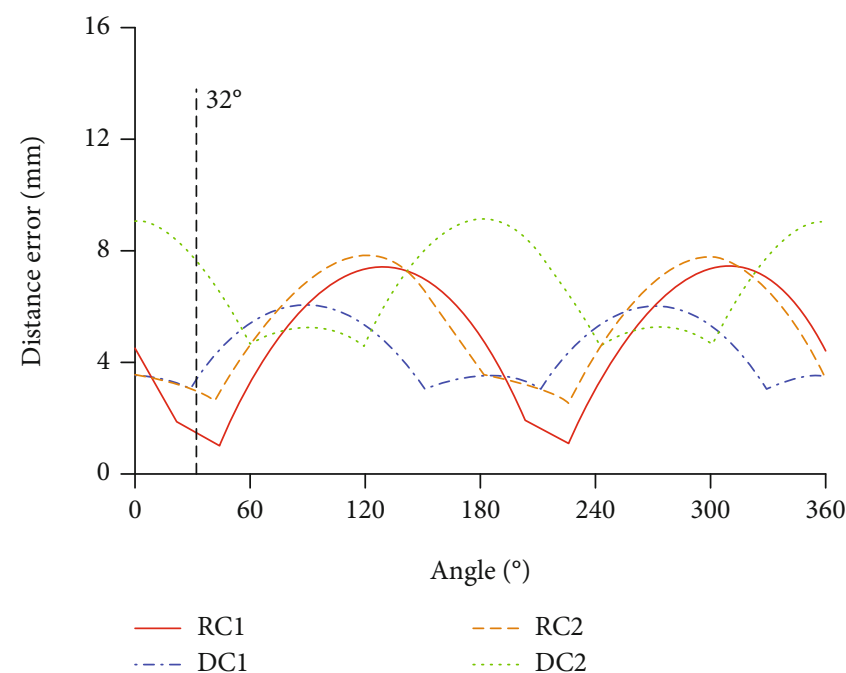

(a)

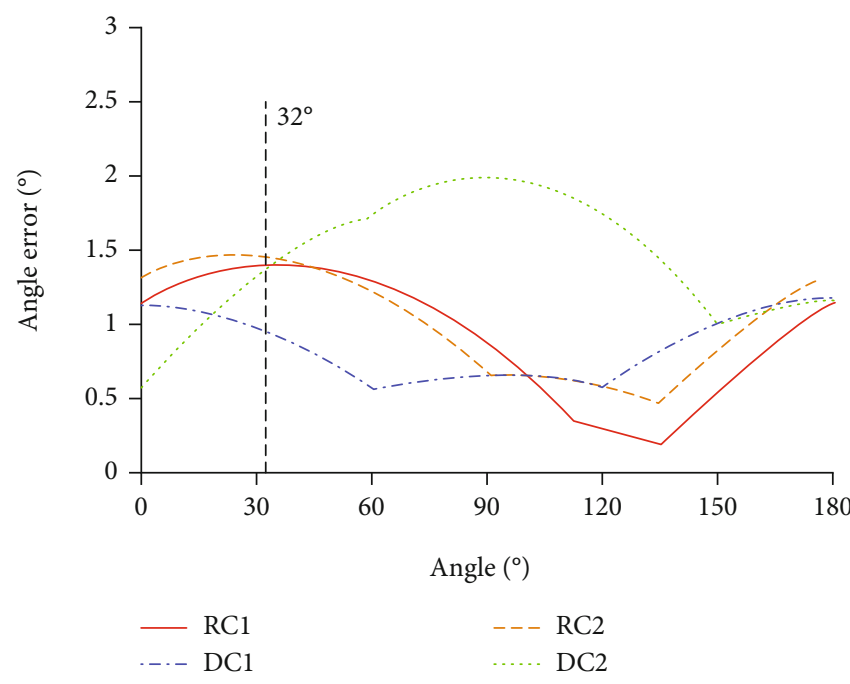

(b)

Figure 9: The distance errors and the angle errors of various rosette configurations. (a) Distance error and (b) Angle error.

TABLE 3: The experimental results of damage localization.

\begin{tabular}{lcccccccc}
\hline Damage number & \multicolumn{3}{c}{ Rosette 1} & \multicolumn{2}{c}{ Rosette 2 } & \multicolumn{2}{c}{ Location } \\
& Actual $\left(^{\circ}\right)$ & Test $\left({ }^{\circ}\right)$ & $\% \mathrm{E}$ & Actual $\left(^{\circ}\right)$ & Test $\left(^{\circ}\right)$ & $\% \mathrm{E}$ & Actual $(\mathrm{mm})$ & Test $(\mathrm{mm})$ \\
\hline Damage 1 & 56.4 & 59.0 & 4.6 & 31.0 & 25.2 & -18.8 & $(350,400)$ & $(391,451)$ \\
Damage 2 & 108.4 & 101.4 & -6.5 & 56.3 & 57.2 & 1.5 & $(450,400)$ & $(486,373)$ \\
\hline
\end{tabular}

between the simulation results and the experimental results are caused by measurement errors and noise.

As presented in Equation (12), the attenuation caused by different distances can generate differences in the wave amplitude detected by each piezoelectric fiber in different rosette configurations, and the actual angle deviates from the ideal value. This deviation is the error in the Lamb wave direction estimation method. As the scatter wave direction is varied, the distance and angle errors also vary. To compare the accuracies of four types of rosette configurations, the average errors $\left(\left|\Delta L_{B}\right|+\left|\Delta L_{C}\right|\right) / 2$ and $\left(\left|\Delta \theta_{B}\right|+\left|\Delta \theta_{C}\right|\right) / 2$ of different rosette configurations are plotted in Figure 9. The distance and angle error ranges of DC2 are larger than those of the other rosette configurations; those of RC1 and RC2 are equivalent, and those of DC1 are slightly better than those of $\mathrm{RC} 1$ and RC2. The largest distance error in the direction of $32^{\circ}$ is associated with DC2, which shows poor performance in estimating the Lamb wave propagation direction, as listed in Table 2. RC2 shows better performance than the other rosette configurations due to its small distance and angle error.

4.2. Damage Localization Results. Considering its strong performance, RC2 is employed to perform damage localization tests. Figure 5(b) shows the arrangement of two rosettes and the actuator. The excitation central frequency is $40 \mathrm{kHz}$. Considering the damage as a secondary wave source actuator, the corresponding damage location can be estimated with the scattered signals. The related scattered wave propagation directions of the two rosettes and the predicted damage locations are listed in Table 3. The intersection point of two direction lines provides the predicted damage location. The predicted and actual damage locations are shown in Figure 10. The predicted locations are not located far from the actual locations. Note that when the damage is on the path between the actuator and the rosette, the damage- 


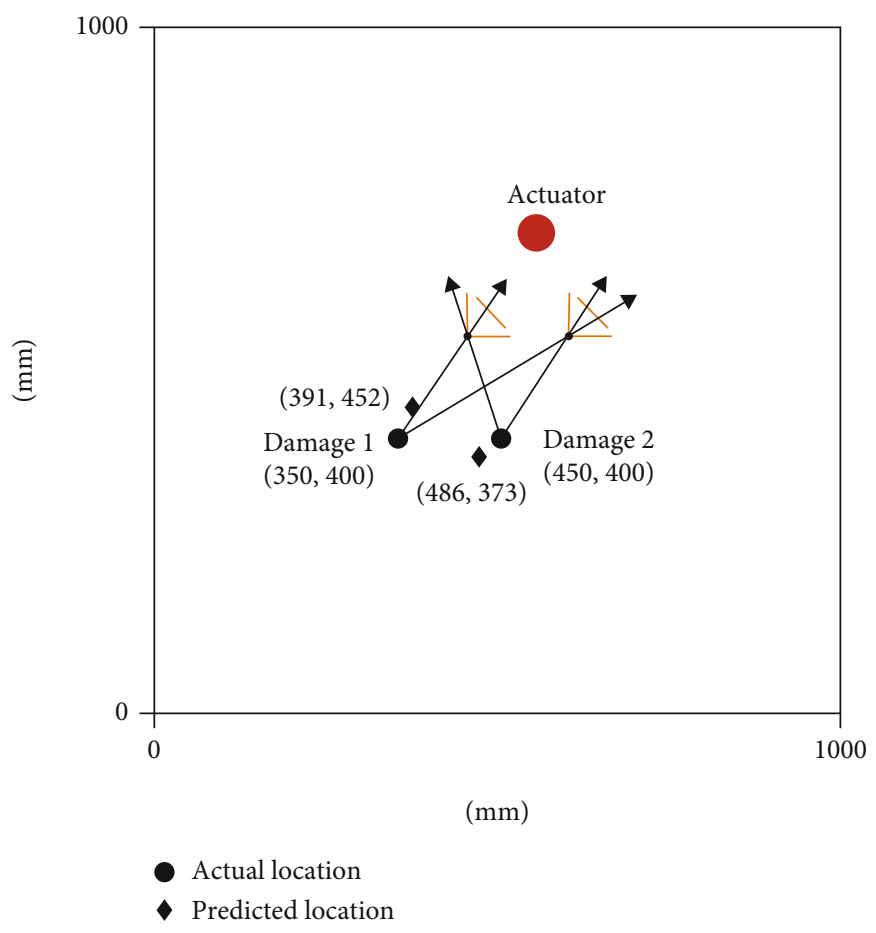

Figure 10: The predicted and actual damage locations.

transmitted wave packets are applied to estimate the scattered wave propagation direction. When the damage is located on or near the line between two rosettes, more rosettes are required to solve for the damage location [10]. In the future, the prediction process can be improved by using transmitted and reflected wave packets from additional rosettes for wave direction estimation.

\section{Conclusion}

This paper focuses on a damage localization method by using two piezoelectric fiber rosettes to measure the scattered Lamb wave propagation direction. The advantage of this method is that wave speed or time-of-flight information is not needed. The effects of various piezoelectric fiber rosette configurations, i.e., $45^{\circ}$-rectangular, $135^{\circ}$-rectangular, $60^{\circ}$-delta, and $120^{\circ}$-delta configurations, on the accuracies of Lamb wave propagation direction estimation are investigated. Mechanical-electric coupled finite element analyses and experimental tests are performed. The MP algorithm that is based on GA optimization by using Hann-window excitation as an atom is proposed to extract the weak damage-related wave packet. The rosette in the $135^{\circ}$-rectangular configuration shows satisfactory performance in determining the wave direction, but the $120^{\circ}$-delta configuration suffers from poor accuracy. Error analyses are performed by analyzing the distance and the angle error of each piezoelectric fiber, which deviates from the theoretical assumption. Considering damage as a secondary wave source, the damage location is determined by the intersection of two scattered wave propagation directions with two rosettes. The proposed damage localization method is validated by experimental tests, and the predicted locations are close to the actual damage locations. Future work will focus on improving the damage localization by using transmitted and reflected wave packets from a larger number of rosettes.

\section{Data Availability}

The data and the MATLAB programs used to support the findings of this study are available from the corresponding author upon request.

\section{Conflicts of Interest}

The authors declare no conflict of interest.

\section{Authors' Contributions}

S.J. contributed to the methodology, experimental test, and writing-original. Y.S. supervised this research and helped in data analysis and modification. S.W. was responsible for the data analysis and the writing-review and editing. Y.P. and Y.L. were responsible for the simulation and validation. All authors have read and agreed to the published version of the manuscript.

\section{Acknowledgments}

This research was funded by the Hunan Innovative Province Construction Special Foundation (No. 2020RC3049), the Hunan Provincial Natural Science Foundation (No. 2020JJ6029), and the Foundation of Hunan Educational Committee (No. 18B567). 


\section{References}

[1] X. Qing, W. Li, Y. Wang, and H. Sun, "Piezoelectric transducer-based structural health monitoring for aircraft applications," Sensors, vol. 19, no. 3, p. 545, 2019.

[2] X. Li, Z. Yang, and X. Chen, "Quantitative damage detection and sparse sensor array optimization of carbon fiber reinforced resin composite laminates for wind turbine blade structural health monitoring," Sensors, vol. 14, no. 4, pp. 73127331, 2014.

[3] T. Stepinski, M. Mańka, and A. Martowicz, "Interdigital lamb wave transducers for applications in structural health monitoring," NDT \& E International, vol. 86, pp. 199-210, 2017.

[4] T. Kundu, H. Nakatani, and N. Takeda, "Acoustic source localization in anisotropic plates," Ultrasonics, vol. 52, no. 6, pp. 740-746, 2012.

[5] X. Lin, G. Chen, J. Li, F. Lu, S. Huang, and X. Cheng, "Investigation of acoustic emission source localization performance on the plate structure using piezoelectric fiber composites," Sensors and Actuators A: Physical, vol. 282, pp. 9-16, 2018.

[6] V. Giurgiutiu, SHM of Aerospace Composites-Challenges and Opportunities, CAMX Conference Proceedings, Dallas, TX, USA, 2015.

[7] D. C. Betz, G. Thursby, B. Culshaw, and W. J. Staszewski, "Lamb wave detection and source location using fiber Bragg gratin rosettes," in Smart Structures and Materials 2003: Smart Sensor Technology and Measurement Systems, vol. 5050, pp. 117-128, San Diego, CA, USA, July 2003.

[8] H. M. Matt and F. L. di Scalea, "Macro-fiber composite piezoelectric rosettes for acoustic source location in complex structures," Smart Materials and Structures, vol. 16, no. 4, pp. 14891499, 2007.

[9] S. Salamone, I. Bartoli, P. di Leo et al., "High-velocity impact location on aircraft panels using macro-fiber composite piezoelectric rosettes," Journal of Intelligent Material Systems and Structures, vol. 21, no. 9, pp. 887-896, 2010.

[10] C. Zhang, J. Qiu, H. Ji, and S. Shan, “An imaging method for impact localization using metal-core piezoelectric fiber rosettes," Journal of Intelligent Material Systems and Structures, vol. 26, no. 16, pp. 2205-2215, 2015.

[11] P. Zhao, D. Pisani, and C. S. Lynch, "Piezoelectric strain sensor/actuator rosettes," Smart Materials and Structures, vol. 20, no. 10, p. 102002, 2011.

[12] S. Wang, W. Wu, Y. Shen, H. Li, and B. Tang, "Lamb wave directional sensing with piezoelectric fiber rosette in structure health monitoring," Shock and Vibration, vol. 2019, Article ID 6189290, 12 pages, 2019.

[13] S. Yin, Z. Cui, and T. Kundu, "Acoustic source localization in anisotropic plates with "Z" shaped sensor clusters," Ultrasonics, vol. 84, pp. 34-37, 2018.

[14] N. Sen and T. Kundu, "Acoustic source localization in a highly anisotropic plate with unknown orientation of its axes of symmetry and material properties with numerical verification," Ultrasonics, vol. 100, article 105977, 2020.

[15] J. Zhao, J. Qiu, H. Ji, and N. Hu, "Four vectors of Lamb waves in composites: semianalysis and numerical simulation," Journal of Intelligent Material Systems and Structures, vol. 24, no. 16, pp. 1985-1994, 2013.

[16] V. Micro-Measurements, Strain Gage Selection: Criteria, Procedures, Recommendations, Technical Note. Vishay Precision Group, Inc. TN-5052007, 2007.
[17] D. A. Drake, R. W. Sullivan, and J. C. Wilson, "Distributed strain sensing from different optical fiber configurations," Inventions, vol. 3, no. 4, p. 67, 2018.

[18] C. Xu, Z. Yang, S. Tian, and X. Chen, "Lamb wave inspection for composite laminates using a combined method of sparse reconstruction and delay-and-sum," Composite Structures, vol. 223, p. 110973, 2019.

[19] Y. Shen and V. Giurgiutiu, "Combined analytical FEM approach for efficient simulation of Lamb wave damage detection," Ultrasonics, vol. 69, pp. 116-128, 2016.

[20] W. Wang, Y. Bao, W. Zhou, and H. Li, "Sparse representation for Lamb-wave-based damage detection using a dictionary algorithm," Ultrasonics, vol. 87, pp. 48-58, 2018.

[21] S. G. Mallat and Z. Zhifeng, "Matching pursuits with timefrequency dictionaries," IEEE Transactions on Signal Processing, vol. 41, no. 12, pp. 3397-3415, 1993.

[22] J. C. Hong, K. H. Sun, and Y. Y. Kim, “The matching pursuit approach based on the modulated Gaussian pulse for efficient guided-wave damage inspection," Smart Materials and Structures, vol. 14, no. 4, pp. 548-560, 2005.

[23] A. Raghavan and C. E. S. Cesnik, "Guided-wave signal processing using chirplet matching pursuits and mode correlation for structural health monitoring," Smart Materials and Structures, vol. 16, no. 2, pp. 355-366, 2007.

[24] H. W. Kim and F. G. Yuan, "Enhanced damage imaging of a metallic plate using matching pursuit algorithm with multiple wavepaths," Ultrasonics, vol. 89, pp. 84-101, 2018.

[25] Y. Xu, M. Luo, Q. Liu, G. du, and G. Song, "PZT transducer array enabled pipeline defect locating based on time-reversal method and matching pursuit de-noising," Smart Materials and Structures, vol. 28, no. 7, article 075019, 2019.

[26] Y. Lu and J. E. Michaels, "Numerical implementation of matching pursuit for the analysis of complex ultrasonic signals," IEEE Transactions on Ultrasonics, Ferroelectrics, and Frequency Control, vol. 55, no. 1, pp. 173-182, 2008.

[27] Y. Liu, C. Shen, Y. Wang, and F. Sun, "Guided wave NDT signal recognition with orthogonal matching pursuit based on modified evolutionary programming," AASRI Procedia, vol. 3, pp. 43-48, 2012.

[28] A. L. Qi, G. M. Zhang, M. Dong, H. W. Ma, and D. M. Harvey, "An artificial bee colony optimization based matching pursuit approach for ultrasonic echo estimation," Ultrasonics, vol. 88, pp. 1-8, 2018. 